Monatsschr Kinderheilkd 2013 · 161:842-842

DOI 10.1007/s00112-013-2977-5

Online publiziert: 8. August 2013

(c) Springer-Verlag Berlin Heidelberg 2013

W. Sauseng ${ }^{1} \cdot$ N. Hofer ${ }^{1} \cdot$ M. Hausegger ${ }^{1} \cdot$ H. Kenzian ${ }^{2} \cdot$ S. Weiss ${ }^{3} \cdot$ R. RathWacenovsky ${ }^{4} \cdot$ R. Kerbl ${ }^{5}$

${ }^{1}$ Univ.-Klinik für Kinder- und Jugendheilkunde, Medizinische Universität Graz

${ }^{2}$ Abteilung für Kinder- und Jugendheilkunde, LKH Villach

${ }^{3}$ Preyer'sches Kinderspital, Wien

${ }^{4}$ Kinder- und Jugendabteilung, Landesklinikum Baden-Mödling, Baden

${ }^{5}$ Abteilung für Kinder und Jugendliche, LKH Leoben

\title{
Zum Konsensuspapier „Medikamentöse Behandlung von Atmungsstörungen bei adenotonsillärer Hyperplasie“
}

\section{Kommentar der Arbeitsgruppe Schlafmedizin und Schlafforschung der Österreichischen Gesellschaft für Kinder- und Jugendheilkunde}

Was OSAS angeht, folgen wir der Empfehlung der American Acadamy of Pediatrics, die die HNO-chirurgische Therapie als primäre Therapie bei OSAS und adenotonsillärer Hyperplasie empfiehlt [2]. Da sowohl durch intranasal-verabreichte Steroide $[3,4]$ als auch für den Leukotrienantagonisten Montelukast [5] eine signifikante Verbesserung der OSAS-Symptomatik nachgewiesen wurde, ohne dass es zum vollständigen Sistieren der Beschwerden kam, können diese Medikamente im „off label use“ als Überbrückung der Zeit zwischen Erstvorstellung, Diagnostik und primär empfohlener Therapie eingesetzt werden.

Ziel muss jedoch aus unserer Sicht auch zur Vermeidung von Sekundärveränderungen - immer die Herstellung einer physiologischen unbeeinträchtigten Atemsituation sein. Die konservative Therapie sollte nicht Anlass dazu geben, eine notwendige chirurgische Intervention unnötig lange zu verzögern.

\section{Korrespondenzadresse}

\section{Dr. W. Sauseng}

Univ.-Klinik für Kinder- und Jugendheilkunde, Medizinische Universität Graz Auenbruggerplatz 34/2, $8036 \mathrm{Graz}$ Österreich

we.sauseng@medunigraz.at

\section{Einhaltung ethischer Richtlinien}

Interessenkonflikt. W. Sauseng, N. Hofer, J. Pansy, M. Hausegger, H. Kenzian, S. Weiss, R. Rath-Wacenovsky, R. Kerbl geben an, dass kein Interessenkonflikt besteht.

Das vorliegende Manuskript enthält keine Studien an Menschen oder Tieren.

\section{Literatur}

1. Kenzian H, Weiss S, Zotter H et al (2011) Schnarchen und obstruktives Schlafapnoesyndrom (OSAS). Monatsschr Kinderheilkd 159:667-670

2. Marcus CL, Brooks LJ, Ward SD et al (2012) Diagnosis and management of childhood obstructive sleep apnea syndrome. Pediatrics 130:e714-755

3. Zhang L, Mendoza-Sassi RA, Cesar JA et al (2008) Intranasal corticosteroids for nasal airway obstruction in children with moderate to severe adenoidal hypertrophy. Cochrane Database Syst Rev:CD006286

4. Kuhle S, Urschitz MS (2011) Anti-inflammatory medications for obstructive sleep apnea in children. Cochrane Database Syst Rev:CD007074

5. Goldbart AD, Greenberg-Dotan S, Tal A (2012) Montelukast for children with obstructive sleep apnea: a double-blind, placebo-controlled study. Pediatrics 130:e575-580 